\title{
A chaperone surveillance system in plant circadian rhythms
}

\author{
Joon-Yung Cha , Laila Khaleda ${ }^{\#}$, Hee Jin Park \& Woe-Yeon Kim ${ }^{*}$ \\ Division of Applied Life Science (BK21Plus), PMBBRC \& IALS, Gyeongsang National University, Jinju 52828, Korea
}

\begin{abstract}
The circadian clock is an internal system that is synchronized by external stimuli, such as light and temperature, and influences various physiological and developmental processes in living organisms. In the model plant Arabidopsis, transcriptional, translational and post-translational processes are interlocked by feedback loops among morning- and eveningphased genes. In a post-translational loop, plant-specific singlegene encoded GIGANTEA (GI) stabilize the F-box protein ZEITLUPE (ZTL), driving the targeted-proteasomal degradation of TIMING OF CAB EXPRESSION 1 (TOC1) and PSEUDORESPONSE REGULATOR 5 (PRR5). Inherent to this, we demonstrate the novel biochemical function of $\mathrm{Gl}$ as a chaperone and/or co-chaperone of Heat-Shock Protein 90 (HSP90). Gl prevents ZTL degradation as a chaperone and facilitates ZTL maturation together with HSP90/HSP70, enhancing ZTL activity in vitro and in planta. GI is known to be involved in a wide range of physiology and development as well as abiotic stress responses in plants, but it could also interact with diverse client proteins to increase protein maturation. Our results provide evidence that GI helps proteostasis of ZTL by acting as a chaperone and a co-chaperone of HSP90 for proper functioning of the Arabidopsis circadian clock. [BMB Reports 2017; 50(5): 235-236]
\end{abstract}

Circadian clock rhythms regulate numerous metabolic, physiological, and developmental responses in living organisms

*Corresponding author. E-mail: kim1312@gnu.ac.kr

${ }^{\text {\#} T h e s e ~ a u t h o r s ~ c o n t r i b u t e d ~ e q u a l l y ~ t o ~ t h i s ~ w o r k . ~}$

https://doi.org/10.5483/BMBRep.2017.50.5.064

Received 15 April 2017

Keywords: Chaperone, Circadian clock, GIGANTEA, HSP90, ZEITLUPE

Abbreviations: CDF1, Cyclic DOF factor 1; COP1, Constitutive photomorphogenesis 1; FKF1, Flavin-binding kelch repeat F-box protein 1; SOS2, Salt overly sensitive 2; SPY, Spindly; SVP, Short vegetative phase; TEM1, Tempranillo 1

Perspective to: Joon-Yung Cha et al (2017), GIGANTEA is a co-chaperone which facilitates maturation of ZEITLUPE in the Arabidopsis circadian clock, Nature Communications, 8(1):3. DOI: 10.1038/s41467-016-0014-9 in relation to the approximately 24-hour light-dark cycle of the Earth. In plants, diurnal and seasonal variations are coupled to changes in day-length and temperature, which serve as input signals. These are transduced to central circadian oscillators in Arabidopsis plants, such as CCA1 (CIRCADIAN CLOCKASSOCIATED 1), LHY (LATE ELONGATED HYPOCOTYL) and TOC1 (TIMING OF CAB EXPRESSION 1). They transcriptionally and post-transcriptionally regulate the expression of various morning-phased and evening-phased genes that interlock via feedback loops (Greenham and McClung (2015) Nat Rev Genet 16, 598-610). In brief, CCA1 and $L H Y$ peaking at dawn activate the morning-phased genes PRR9 (PSEUDO-RESPONSE REGULATOR 9) and PRR7, which repress CCA1 and LHY. The PRRs negatively regulate the expression of RVE8 (REVEILLE 8), which activates evening-phased genes, such as TOC1, LUX (LUX ARRHYTHMO) and ELF4 (EARLY FLOWERING 4). Evening complex (EC) including ELF3, ELF4 and LUX repress $P R R 9$ and PRR7. Interestingly, post-translational regulation in plant circadian clock rhythms also involves the proteasomal degradation of TOC1 and PRR5 through the F-box protein ZTL (ZEITLUPE) that possesses a blue light-sensing LOV (Light, Oxygen, Voltage) domain. ZTL proteins oscillate diurnally with evening-phased peaks occurring in the absence of transcriptional changes. ZTL protein abundance is determined by the vascular, plant-specific, single-gene-encoded protein Gl (GIGANTEA), which displays circadian oscillations in both mRNA and protein levels (Kim et al (2007) Nature 449, 356-360). The absence of ZTL or GI in Arabidopsis lengthens the period of rhythms by approximately $2 \mathrm{~h}$. Thus, the accumulation of GI proteins helps to stabilize ZTL proteins to enable proper functioning of the circadian clock.

Molecular chaperones not only inhibit protein denaturation and aggregation but also facilitate protein folding and refolding. HSP90 (HEAT-SHOCK PROTEIN 90)-associated heteromeric chaperone machinery is well characterized in animal cells, and is composed by HSP90, HSP70, HOP (HSP90/HSP70 ORGANIZING PROTEIN), HSP40 and p23. This captures denatured substrates, and refolds the substrates with correctly folded states, thereby activating enzyme activity. We have reported that the diurnal rhythmicity of Arabidopsis circadian clocks is lengthened and that ZTL protein levels are diminished when cytosolic HSP90s are absent or deactivated by the Hsp90 inhibitor geldanamycin (Kim et al (2011) Proc Natl Acad Sci U S A 108, 16843-16848). Our results showed that the molecular chaperone activity of HSP90 is necessary

ISSN: 1976-670X (electronic edition)

Copyright (c) 2017 by the The Korean Society for Biochemistry and Molecular Biology

(c) This is an open-access article distributed under the terms of the Creative Commons Attribution Non-Commercial License (http://creativecommons.org/licenses/by-nc/4.0) which permits unrestricted non-commercial use, distribution, and reproduction in any medium, provided the original work is properly cited. 


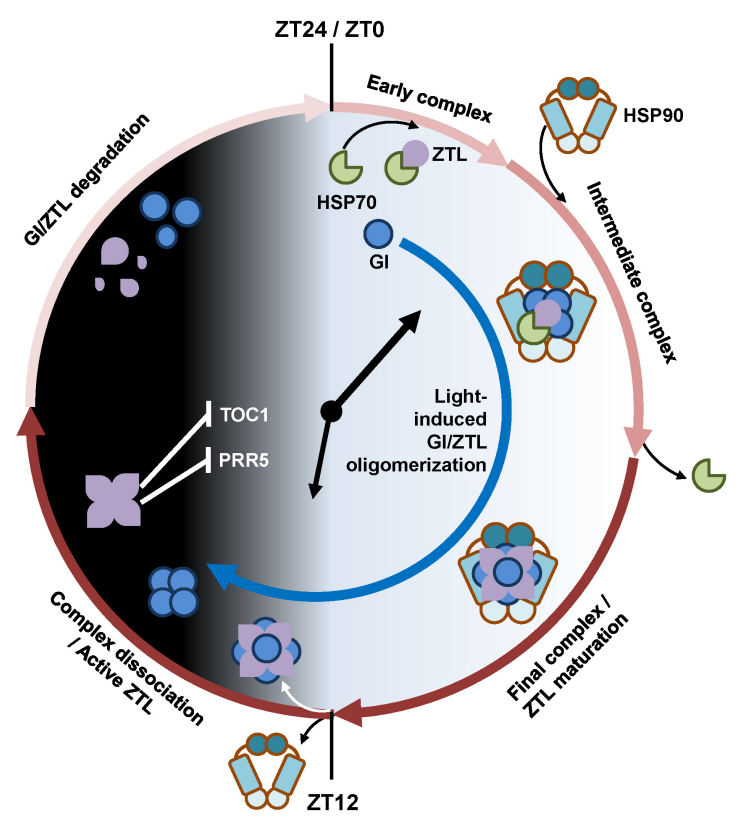

Fig. 1. Chaperone machinery composed of HSP90/HSP70/GI/ZTL in plant circadian rhythms. The blue light-sensing F-box protein ZTL oscillates with day-time accumulation and night-time degradation in a post-translational loop. Nascent ZTL polypeptide may be captured by HSP70 in an early complex and then transferred to HSP90-Gl to form an intermediate complex. Gl as a cochaperone of HSP90 helps ZTL maturation to activate proteasomal degradation of PRR5 and TOC1 at night.

for proper folding of ZTL proteins. Based on these findings, it has been questioned whether the possible function of $\mathrm{Gl}$ as a chaperone that maintains the circadian clock in Arabidopsis is to protect ZTL proteins or to enhance the folding of ZTL with HSP90.

Our study demonstrates that Gl exhibits novel chaperone activity that protects and enhances the refolding of model substrates (such as malate dehydrogenase and glucose-6phosphate dehydrogenase) as well as that of real substrate ZTL, in vitro (Fig. 1). The diurnal oscillation of ZTL proteins without transcriptional regulation is essential for the accumulation of a necessary amount of ZTL proteins and to function as an E3 ligase-degrading TOC1 and PRR5, enabling the proper functioning of the circadian clock. The protein abundance and enzyme activity of ZTL are regulated by proteostasis. HSP90based chaperone machinery is composed by various cochaperones such as HOP, AHA (ACTIVATOR OF HSP90 ATPASE), SGT (SUPPRESSOR OF G2 ALLELE of SKP1) and p23, which regulate the ATPase activity of HSP90, positively or negatively. We also found that Gl synergistically enhances HSP90/HSP70 activity for the maturation of ZTL. This suggests that $\mathrm{Gl}$ serves as a co-chaperone that positively regulates the HSP90 chaperone and promotes ZTL maturation to play as a F-box E3 ligase for the degradation of TOC1 and PRR5. Consistent with in planta results, the accumulation or the activity of ZTL-LUC is determined by GI protein abundance. Thus, the oscillation of ZTL proteins are regulated by both $\mathrm{Gl}$ and HSP90, indicating that Gl may protect ZTL protein as a chaperone and/or help the maturation of ZTL protein as a co-chaperone along with the major molecular chaperone HSP90.

$\mathrm{Gl}$ is a large and unique plant protein that is not known to contain a conserved domain, but that is involved in diverse physiological and developmental processes such as flowering (FKF1, CDF1, SVP, TEM1, TEM2, COP1 and ELF3 as partner proteins), circadian clock (ZTL and TOC1), light signaling, hypocotyl elongation (SPY), sucrose signaling, starch accumulation, chlorophyll accumulation, transpiration, and salt stress response (SOS2) (Mishra and Paniqrahi (2015) Front Plant Sci 6, 8). However, the ways that Gl regulates this wide range of physiological processes in plants still needs to be elucidated. Our findings reveal the novel Gl biochemical functions of acting as a chaperone and/or co-chaperone that can affect the maturation of large numbers of client proteins that regulate diverse physiological processes.

\section{ACKNOWLEDGEMENTS}

This work was supported by a grant from the Cooperative Research Program for Agriculture, Science, \& Technology Development (project no. PJ010953042017), Rural Development Administration, Republic of Korea (WYK). 